\title{
Longitudinal dynamics and chiral symmetry breaking in holographic light-front QCD
}

\author{
Guy F. de Téramond $\oplus^{1, *}$ and Stanley J. Brodsky $\oplus^{2, \dagger}$ \\ ${ }^{1}$ Laboratorio de Física Teórica y Computacional, Universidad de Costa Rica, 11501 San José, Costa Rica \\ ${ }^{2}$ SLAC National Accelerator Laboratory, Stanford University, Stanford, California 94309, USA
}

(Received 21 March 2021; revised 27 May 2021; accepted 3 November 2021; published 16 December 2021)

\begin{abstract}
The breaking of chiral symmetry in holographic light-front QCD is encoded in its longitudinal dynamics, with its chiral limit protected by the superconformal algebraic structure which governs its transverse dynamics. The scale in the longitudinal light-front Hamiltonian determines the confinement strength in this direction: It is also responsible for most of the light meson ground state mass consistent with the Gell-Mann-Oakes-Renner constraint. Longitudinal confinement and the breaking of chiral symmetry are found to be different manifestations of the same underlying dynamics as found in the 't Hooft large- $N_{C}$ QCD $(1+1)$ model.
\end{abstract}

DOI: 10.1103/PhysRevD.104.116009

\section{INTRODUCTION}

In spite of the important progress of Euclidean lattice gauge theory, a basic understanding of the mechanism of color confinement and its relation to chiral symmetry breaking in QCD, two fundamental phenomena of strong interactions, has remained an unsolved problem. Recent developments based on superconformal quantum mechanics [1,2] in light-front quantization [3] and its holographic embedding on a higher dimensional gravity theory [4] (gauge/gravity correspondence) have led to new analytic insights into the structure of hadrons and their dynamics [5-10]. This new approach to nonperturbative QCD dynamics, holographic light-front QCD, leads to effective semiclassical relativistic bound-state equations for arbitrary spin [11], and it incorporates fundamental properties which are not apparent from the QCD Lagrangian, such as the emergence of the hadron mass scale, the prediction of a massless pion in the chiral limit, and the remarkable connections between meson, baryon and tetraquark spectroscopy across the full hadron spectrum [12-15]. Phenomenological extensions of the holographic QCD approach provide nontrivial connections between the dynamics of form factors and polarized and unpolarized quark distributions with pre-QCD nonperturbative approaches such as Regge theory and the Veneziano

\footnotetext{
guy.deteramond@ucr.ac.cr

sjbth@slac.stanford.edu
}

Published by the American Physical Society under the terms of the Creative Commons Attribution 4.0 International license. Further distribution of this work must maintain attribution to the author(s) and the published article's title, journal citation, and DOI. Funded by SCOAP ${ }^{3}$. model [16-18], and also describe the running of the QCD coupling $\alpha_{s}\left(Q^{2}\right)$ in the nonperturbative domain [19,20].

In this article we examine the effect of longitudinal lightfront (LF) dynamics for the computation of hadron masses, confinement, and chiral symmetry breaking motivated by the previous work in Refs. [21-30]. Although the semiclassical model approximation to LF quantized QCD determines the confinement potential in the LF transverse coordinates in the zero quark mass limit [10], an extension is required which incorporates color-confining LF longitudinal dynamics for nonzero quark masses [31]. This extension of holographic LF QCD (HLFQCD) should preserve its successful predictions and incorporate chiral symmetry breaking, while restoring 3-dimensional rotational invariance in the heavy-quark limit.

The main contents of this article are as follows. In Sec. II we briefly review the extension of the holographic lightfront framework to include quark masses by combining the longitudinal dynamics with the HLFQCD transverse dynamics by following [27,30]. In Sec. III we recall how the transverse confinement potentials for mesons, baryons and tetraquarks are determined by the underlying conformal symmetry of light-front QCD and its embedding in AdS space [10]. We further examine in this section two models of longitudinal confinement: The 't Hooft large- $N_{C}$ model in one-space and one time-time dimension, as the prototypical example of a simple model derived from QCD, which allows the computation of a meson spectrum while incorporating chiral symmetry breaking. We also examine in this section the longitudinal potential model introduced in [30], which generates a complete basis function and leads to important constraints in the large quark mass nonrelativist limit. These aspects, together with the HLFQCD constraints, are used in Sec. IV to extend our 
previous superconformal holographic results $[9,10]$ to include longitudinal dynamics and chiral symmetry breaking in the semiclassical Hamiltonian light-front wave equations. Numerical results are presented in Sec. V, including a computation of meson masses and distribution amplitudes. Some final comments are included in Sec. VI.

\section{LONGITUDINAL AND TRANSVERSE DYNAMICS IN HLFQCD}

We start with the light-front invariant QCD Hamiltonian

$$
\begin{gathered}
H^{L F}=P^{2}=P^{+} P^{-}-\mathbf{P}_{\perp}^{2}, P^{2}=P_{\mu} P^{\mu}=M^{2}, \\
H^{L F}|\psi\rangle=M^{2}|\psi\rangle,
\end{gathered}
$$

and compute $M^{2}$ from the hadronic matrix element $\left\langle\psi\left(P^{\prime}\right)\left|H^{L F}\right| \psi(P)\right\rangle=M^{2}\left\langle\psi\left(P^{\prime}\right) \mid \psi(P)\right\rangle$, expanding the hadronic states in terms of their Fock components [5,9]. For the simplest hadronic bound-state, a two parton state with quark masses $m_{q}$ and $m_{\bar{q}}$,

$$
\begin{aligned}
M^{2}= & \int_{0}^{1} d x \int \frac{d^{2} \mathbf{k}_{\perp}}{16 \pi^{3}}\left(\frac{\mathbf{k}_{\perp}^{2}}{x(1-x)}+\frac{m_{q}^{2}}{x}+\frac{m_{\bar{q}}^{2}}{(1-x)}\right)\left|\psi\left(x, \mathbf{k}_{\perp}\right)\right|^{2} \\
& +\int_{0}^{1} d x \int \frac{d^{2} \mathbf{k}_{\perp}}{16 \pi^{3}} U\left(x, \mathbf{k}_{\perp}\right)\left|\psi\left(x, \mathbf{k}_{\perp}\right)\right|^{2}
\end{aligned}
$$

with normalization

$$
\int_{0}^{1} d x \int \frac{d^{2} \mathbf{k}_{\perp}}{16 \pi^{3}}\left|\psi\left(x, \mathbf{k}_{\perp}\right)\right|^{2}=1 .
$$

The variable $x$ is the LF longitudinal momentum fraction $x=k^{+} / P^{+}$and $\mathbf{k}_{\perp}$ is the relative transverse momentum. Taking the Fourier transform, Eq. (2) can be expressed in terms of the transverse impact variable $\mathbf{b}_{\perp}$, conjugate to $\mathbf{k}_{\perp}$,

$$
\begin{aligned}
M^{2}= & \int_{0}^{1} d x \int d^{2} \mathbf{b}_{\perp} \psi^{*}\left(x, \mathbf{b}_{\perp}\right) \\
& \times\left(\frac{-\nabla_{\mathbf{b}_{\perp}}^{2}}{x(1-x)}+\frac{m_{q}^{2}}{x}+\frac{m_{\bar{q}}^{2}}{(1-x)}\right) \psi\left(x, \mathbf{b}_{\perp}\right) \\
& +\int_{0}^{1} d x \int d^{2} \mathbf{b}_{\perp} \psi^{*}\left(x, \mathbf{b}_{\perp}\right) U\left(x, \mathbf{b}_{\perp}\right) \psi\left(x, \mathbf{b}_{\perp}\right),
\end{aligned}
$$

with normalization

$$
\int_{0}^{1} d x \int d^{2} \mathbf{b}_{\perp}\left|\psi\left(x, \mathbf{b}_{\perp}\right)\right|^{2}=1 .
$$

As noted in Refs. [27,30], the LF Hamiltonian equation (1) leads to a longitudinal and a transverse light-front wave equation in the approximation where transverse and longitudinal dynamics are separated. To show this, we introduce the invariant LF transverse variable $\zeta^{2}=x(1-x) \mathbf{b}_{\perp}^{2}$, which is identified with the holographic AdS variable $z$ [9], and factor out the longitudinal, transverse and orbital dependence of the LF wave function [5,9]

$$
\psi(x, \zeta, \varphi)=e^{i L \varphi} X(x) \frac{\phi(\zeta)}{\sqrt{2 \pi \zeta}},
$$

in the approximation where the effective LF confinement potential is written as the sum of longitudinal and transverse components $[27,30]$

$$
U(x, \zeta)=U_{\perp}(\zeta)+U_{\|}(x) .
$$

The LF wave function normalization (5) is equivalent to

$$
\begin{aligned}
& \int_{0}^{\infty} d \zeta \phi^{2}(\zeta)=1 \\
& \int_{0}^{1} d x \frac{X^{2}(x)}{x(1-x)}=1
\end{aligned}
$$

Following the procedure introduced in [5], we obtain for (4)

$$
\begin{aligned}
M^{2}= & \int_{0}^{\infty} d \zeta \phi^{*}(\zeta) \sqrt{\zeta}\left(-\frac{d^{2}}{d \zeta^{2}}-\frac{1}{\zeta} \frac{d}{d \zeta}+\frac{L^{2}}{\zeta^{2}}+U_{\perp}(\zeta)\right) \frac{\phi(\zeta)}{\sqrt{\zeta}} \\
& +\int_{0}^{1} d x \chi^{*}(x)\left(\frac{m_{q}^{2}}{x}+\frac{m_{\bar{q}}^{2}}{(1-x)}+U_{\|}(x)\right) \chi(x),
\end{aligned}
$$

where $L$ is the relative LF orbital angular momentum $L \equiv$ $\left|L^{z}\right|_{\max }$ and $X(x)=\sqrt{x(1-x)} \chi(x)$, with the normalization of $\chi(x)$ determined from (9)

$$
\int_{0}^{1} d x \chi^{2}(x)=1 .
$$

In the chiral limit $X(x) \rightarrow \sqrt{x(1-x)}$ [32], and therefore $\chi(x)=1$ for zero quark masses.

Equation (10) is thus equivalent to the semiclassical LF transverse [5,9] and longitudinal [27,30] wave equations for mesons

$$
\left(-\frac{d^{2}}{d \zeta^{2}}-\frac{1-4 L^{2}}{4 \zeta^{2}}+U_{\perp}(\zeta)\right) \phi(\zeta)=M_{\perp}^{2} \phi(\zeta),
$$

$$
\left(\frac{m_{q}^{2}}{x}+\frac{m_{\bar{q}}^{2}}{1-x}+U_{\|}(x)\right) \chi(x)=M_{\|}^{2} \chi(x),
$$

with $M^{2}=M_{\perp}^{2}+M_{\|}^{2}$. Both Eqs. (12) and (13) are relativistic and frame independent. We note that the separation of longitudinal and transverse equations (12) and (13) is natural in the light-front, since the quark masses are associated with the longitudinal component of the kinetic energy, not the transverse part [27]. 
As noted in Refs. [27,30], the longitudinal eigenvalue equation (13) for the longitudinal mass $M_{\|}^{2}$ can be combined with the holographic LF transverse equation (12) for $M_{\perp}^{2}$ to incorporate massive quarks. The longitudinal mass $M_{\|}^{\frac{1}{2}}$ appears as a separation constant in the transverse equation (12), namely $M_{\perp}^{2} \rightarrow M^{2}-M_{\|}^{2}$ [27]. As a result, the structure of the superconformal equation in the transverse direction is not modified, even by heavy quark masses, as long as transverse and longitudinal dynamics can be separated.

\section{A. HLFQCD equations for baryons}

The semiclassical approximation to the light-front Hamiltonian for baryons is given by a system of two coupled linear differential equations for the chiral components $\psi_{+}$and $\psi_{-}$, which follow from the mapping of higher-dimensional wave equations for half-integer spin in AdS space to the light-front $[9,11]$

$$
\begin{gathered}
-\frac{d}{d \zeta} \psi_{-}-\frac{L+\frac{1}{2}}{\zeta} \psi_{-}-V(\zeta) \psi_{-}=M \psi_{+}, \\
\frac{d}{d \zeta} \psi_{+}-\frac{L+\frac{1}{2}}{\zeta} \psi_{+}-V(\zeta) \psi_{+}=M \psi_{-},
\end{gathered}
$$

with normalization

$$
\int_{0}^{\infty} d \zeta \psi_{+}^{2}(\zeta)=\int_{0}^{\infty} d \zeta \psi_{-}^{2}(\zeta)=1
$$

The system of linear equations (14) and (15) is equivalent to the system of second order light-front wave equations

$$
\begin{aligned}
& \left(-\frac{d^{2}}{d \zeta^{2}}-\frac{1-4 L^{2}}{4 \zeta^{2}}+U_{\perp}^{+}(\zeta)\right) \psi_{+}(\zeta)=M_{\perp}^{2} \psi_{+}(\zeta) \\
& \left(-\frac{d^{2}}{d \zeta^{2}}-\frac{1-4(L+1)^{2}}{4 \zeta^{2}}+U_{\perp}^{-}(\zeta)\right) \psi_{-}(\zeta)=M_{\perp}^{2} \psi_{-}(\zeta)
\end{aligned}
$$

where

$$
U^{ \pm}(\zeta)=V^{2}(\zeta) \pm V^{\prime}(z)+\frac{1+2 L}{\zeta} V(\zeta)
$$

It corresponds to LF angular momentum $L$ and $L+1$.

\section{B. Invariant mass ansatz}

A simple ansatz to account for quark masses in HLFQCD was introduced in [26] based on the arbitrary off-shell dependence of the LF wave function on the invariant mass squared: It controls the bound state according to the uncertainty principle in quantum mechanics. For a two-parton state this amounts to the substitution

$$
\frac{\mathbf{k}_{\perp}^{2}}{x(1-x)} \rightarrow \frac{\mathbf{k}_{\perp}^{2}}{x(1-x)}+\frac{m_{q}^{2}}{x}+\frac{m_{\bar{q}}^{2}}{1-x},
$$

in the ground-state Gaussian wave function to include the expression for the LF kinetic energy with quark masses: It is also the invariant mass squared $s=\left(p_{q}+p_{\bar{q}}\right)^{2}$ of the $q \bar{q}$ pair. This substitution leads, upon exponentiation, to the longitudinal LF wave function (LFWF) [26]

$$
\chi_{I M}(x)=\mathcal{N} \exp \left(-\frac{1}{2 \lambda}\left[\frac{m_{q}^{2}}{x}+\frac{m_{\bar{q}}^{2}}{1-x}\right]\right),
$$

and thus to a natural factorization of the longitudinal and transverse components of the LFWF, independent of the actual value of the effective quark masses. The label IM stands for invariant mass, and $\mathcal{N}$ is a normalization factor [33]. On the other hand it was shown in [28] that the ansatz

$$
\chi_{\mathrm{CSB}}(x) \sim x^{a}(1-x)^{b},
$$

obtained in [21,22], leads to explicit charge symmetry breaking (CSB) in holographic QCD models. The partonic mass shift contribution to hadron masses [34],

$$
\Delta M^{2}=\int_{0}^{1} d x \chi(x)\left[\frac{m_{q}^{2}}{x}+\frac{m_{\bar{q}}^{2}}{1-x}\right] \chi(x),
$$

used in [26,28] does not incorporate, however, the explicit contribution from a longitudinal potential to hadron masses required to satisfy the virial theorem.

\section{LONGITUDINAL AND TRANSVERSE EFFECTIVE CONFINEMENT POTENTIALS}

We briefly describe in this section the transverse confinement potential for mesons in HLFQCD and examine two particularly interesting models for longitudinal confinement: the well known 't Hooft model [21] in one-space and one-time directions, derived from first principles QCD in the large $N_{C}$ limit, and the effective potential model introduced by Li, Maris, Zhao and Vary (LMZV) [30], which generates a complete basis function in the longitudinal direction.

\section{A. Transverse confinement potential}

The transverse LF equation (12) has the same structure as the wave equations derived in AdS provided that one identifies $\zeta=z$ [5], the holographic fifth-dimensional coordinate of AdS. This precise mapping allows us to relate the LF confinement potential $U_{\perp}$ to the dilaton profile which modifies AdS space [9]. Conformal algebra underlies in LF holography the scale invariance of the QCD Lagrangian [6]. It leads to the introduction of a scale $\lambda$ and harmonic confinement, $U \sim \lambda \zeta^{2}$, maintaining the action conformal invariant $[1,6]$. The oscillator potential 
corresponds to a quadratic dilaton profile and thus to the emergence of linear Regge trajectories [35].

Extension to superconformal algebra leads to a specific connection between mesons, baryons and tetraquarks $[7,8,36]$ underlying the $S U(3)_{C}$ representation properties, since a diquark cluster can be in the same color representation as an antiquark, namely $\overline{3} \in 3 \times 3$. The meson wave function $\phi_{M}$, the upper and lower components of the baryon wave function, $\phi_{B \pm} \equiv \psi_{ \pm}$, and the tetraquark wave function, $\phi_{T}$, can be arranged as a supersymmetric 4-plet matrix $[36,37]$

$$
|\Phi\rangle=\left(\begin{array}{cc}
\phi_{M}^{(L+1)} & \phi_{B-}^{(L+1)} \\
\phi_{B+}^{(L)} & \phi_{T}^{(L)}
\end{array}\right),
$$

with $H_{\perp}^{L F}|\Phi\rangle=M_{\perp}^{2}|\Phi\rangle$. The constraints from superconformal structure uniquely determine the form of the effective transverse confining potential for mesons, nucleons and tetraquarks $[7,8,36]$, including critical constant terms:

$$
\begin{gathered}
U_{M \perp}(\zeta)=\lambda^{2} \zeta^{2}+2 \lambda\left(L_{M}-1\right), \\
U_{B \perp}^{+}(\zeta)=\lambda^{2} \zeta^{2}+2 \lambda\left(L_{B}+1\right), \\
U_{B \perp}^{-}(\zeta)=\lambda^{2} \zeta^{2}+2 \lambda L_{B} \\
U_{T \perp}(\zeta)=\lambda^{2} \zeta^{2}+2 \lambda\left(L_{T}+1\right),
\end{gathered}
$$

and lead to the remarkable relations $L_{M}=L_{B}+1$, $L_{T}=L_{B}$. The superconformal algebra also requires the universality of Regge slopes with a unique scale $\lambda$ for all hadron families, a well known fact in hadron physics, which holds to a good approximation.

The solution of the AdS wave equations for mesons and vector mesons does incorporate the dependence on the total quark spin, $S=0$ for the $\pi$ Regge trajectory and $S=1$ for the $\rho$ trajectory: It is given by the additional term $2 \lambda S$, $S=0,1$, in the LF Hamiltonian. It leads, for example to the correct prediction for the $\pi-\rho$ mass gap: $M_{\rho}^{2}-M_{\pi}^{2}=2 \lambda$. In contrast, for baryons the quark spin interaction within a 2-quark cluster is not contained in the AdS action. To describe the quark spin-spin interaction, which distinguishes for example the nucleons from $\Delta$ particles, we also include an identical term, $2 \lambda S, S=0,1$ in the LF baryon Hamiltonian to maintain hadronic supersymmetry. The final expression for the transverse mass spectrum for mesons, baryons and tetraquarks is given by [36]

$$
\begin{gathered}
M_{M \perp}^{2}=4 \lambda\left(n+L_{M}\right)+2 \lambda S, \\
M_{B \perp}^{2}=4 \lambda\left(n+L_{B}+1\right)+2 \lambda S, \\
M_{T \perp}^{2}=4 \lambda\left(n+L_{T}+1\right)+2 \lambda S,
\end{gathered}
$$

with the same slope in $L$ and $n$, the radial quantum number.

\section{B. The 't Hooft model}

As discussed by Chabysheva and Hiller [27], it is natural to identify the potential for longitudinal dynamics with the potential which underlies the 't Hooft model for large- $N_{C}$ QCD in $(1+1)$ dimensions. It has the same form as the instantaneous LF potential which appears from instantaneous gluon exchange in the $A^{+}=0$ light-cone gauge in QCD $(3+1)$ [38].

In the 't Hooft model [21] the longitudinal equation (13) becomes the integral equation for the pion

$$
\begin{aligned}
& \left(\frac{m_{q}^{2}}{x}+\frac{m_{\bar{q}}^{2}}{1-x}\right) \chi(x)+\frac{g^{2} N_{C}}{\pi} P \int_{0}^{1} d x^{\prime} \frac{\chi(x)-\chi\left(x^{\prime}\right)}{\left(x-x^{\prime}\right)^{2}} \\
& \quad=M_{\|}^{2} \chi(x),
\end{aligned}
$$

where the coupling $g$ has dimensions of mass. To find an analytic solution to (32) the approximate solution

$$
\chi(x) \sim x^{\beta_{1}}(1-x)^{\beta_{2}},
$$

is chosen to cancel the endpoint singularities [21]. Expanding (32) near $x=\epsilon$ we find

$$
\left[\frac{\pi m_{q}^{2}}{g^{2} N_{C}}-1+\pi \beta_{1} \cot \left(\pi \beta_{1}\right)\right] \epsilon^{\beta_{1}-1}=0 .
$$

It leads to the result $\beta_{1}=\left(3 m_{q}^{2} / \pi g^{2} N_{C}\right)^{1 / 2}$ from the expansion of the transcendental equation (34) for $m_{q}^{2} / g^{2} N_{C} \ll 1$. Likewise, we obtain $\beta_{2}=\left(3 m_{\bar{q}}^{2} / \pi g^{2} N_{C}\right)^{1 / 2}$ from the expansion of (32) at the upper bound $x=1-\epsilon$.

Integrating (32)

$$
\begin{aligned}
M_{\|}^{2}= & \int_{0}^{1} d x\left(\frac{m_{q}^{2}}{x}+\frac{m_{\bar{q}}^{2}}{1-x}\right) \chi^{2}(x) \\
& +\frac{g^{2} N_{C}}{\pi} P \int_{0}^{1} d x \int_{0}^{1} d x^{\prime} \frac{\chi(x)\left[\chi(x)-\chi\left(x^{\prime}\right)\right]}{\left(x-x^{\prime}\right)^{2}},
\end{aligned}
$$

and using the approximate solution (33) in the limit of small quark masses we find for the pion mass

$$
M_{\pi}^{2}=g \sqrt{\frac{\pi N_{C}}{3}}\left(m_{q}+m_{\bar{q}}\right)+\mathcal{O}\left(\left(m_{q}+m_{\bar{q}}\right)^{2}\right),
$$

using the Hellmann-Feynman theorem to evaluate the second integral in (35). It has the same linear dependence in the quark mass as the Gell-Mann-Oakes-Renner (GMOR) relation [39]. Spontaneous chiral symmetry breaking in the 't Hooft model occurs in the limit $N_{C} \rightarrow \infty$ followed by the limit $m_{q} \rightarrow 0[21,23]$. 


\section{The LZMV longitudinal potential}

The effective LMZV potential [30]

$$
U_{\|}(x)=-\sigma^{2} \frac{d}{d x}\left(x(1-x) \frac{d}{d x}\right)
$$

was introduced in the context of basis light-front quantization (BLFQ) $[40,41]$. It generates a complete basis of orthonormal functions from the solution of (13)

$$
\chi_{\kappa}^{\alpha, \beta}(x)=N x^{\alpha / 2}(1-x)^{\beta / 2} P_{\kappa}^{(\alpha, \beta)}(1-2 x),
$$

where $\alpha=2 m_{q} / \sigma, \beta=2 m_{\bar{q}} / \sigma, P_{\kappa}^{(\alpha, \beta)}$ is a Jacobi polynomial of order $\kappa$ and $N$ is a normalization factor (Appendix A). The longitudinal basis can be combined with the holographic LFWF basis in transverse space [9] to perform extensive numerical computations using the BLFQ methods [42-44], as well other computations which require a complete basis function [45], including numerical evaluation in quantum computers [46].

The potential (37) leads to an oscillator in the longitudinal dimension which is combined with the transverse oscillator for heavy quark masses $m_{q}, m_{\bar{q}} \rightarrow m_{Q}, m_{\bar{Q}}$ [30]. The recovery of the rotational invariance of the oscillator in the nonrelativistic limit leads to a precise connection of the dimensionful longitudinal coupling $\sigma$ to the transverse scale $\lambda$ (Appendix B)

$$
\sigma=\frac{\lambda_{Q}}{m_{Q}+m_{\bar{Q}}}
$$

where $\lambda_{Q}$ depends on the heavy quark mass $m_{Q}, \lambda \rightarrow \lambda_{Q}$, to keep the ratio (39) constant in the heavy quark mass domain (See Sec. IV B). More relevant for our present purposes, in addition to its simplicity, is the observation that in the limit of small quark masses, the potential (37) leads to the approximate solution (36) obtained in [21,22], thereby incorporating the breaking of chiral symmetry in the LF holographic model consistent with the virial theorem.

\section{EXTENDED HLFQCD MODEL}

The extension of HLFQCD to include quark masses described in this article combines the transverse and longitudinal dynamics as in [27,30], but keeping the physically motivated invariant mass ansatz, which is required in order to incorporate the invariant off-massshell dependence of the full LFWF as in our previous work. In practice, we include the longitudinal dynamics by performing an expansion of the invariant mass LFWF (21) using the convenient basis of complete orthonormal eigenfunctions generated by the specific potential (37)

$$
\begin{aligned}
\chi_{I M}(x) & =\mathcal{N} \exp \left(-\frac{\sigma^{2}}{8 \lambda}\left[\frac{\alpha^{2}}{x}+\frac{\beta^{2}}{1-x}\right]\right) \\
& =\sum_{\kappa} C_{\kappa} \chi_{\kappa}(x) .
\end{aligned}
$$

The expansion coefficients $C_{\kappa}$ are computed from the overlap

$$
C_{\kappa}^{\alpha, \beta}=\int_{0}^{1} d x \chi_{\kappa}^{\alpha, \beta}(x) \chi_{I M}(x)
$$

with $\left\langle\chi_{I M} \mid \chi_{I M}\right\rangle=\sum_{\kappa} C_{\kappa}^{2}=1$. A rapid convergence of the invariant mass LFWF was obtained in [27,30] using the basis wave function (38), with the lowest-index Jacobi polynomial giving the dominant contribution.

In terms of (38) the longitudinal contribution to a meson mass is given by

$$
\begin{aligned}
M_{\|}^{2} / \sigma^{2}= & \int_{0}^{1} d x \chi_{I M}(x)\left(-\frac{d}{d x}\left(x(1-x) \frac{d}{d x}\right)\right. \\
& \left.+\frac{1}{4}\left[\frac{\alpha^{2}}{x}+\frac{\beta^{2}}{1-x}\right]\right) \chi_{I M}(x) \\
= & \sum_{\kappa} C_{\kappa}^{2} \nu^{2}(\kappa, \alpha, \beta),
\end{aligned}
$$

expressed of a sum of the eigenvalues

$$
\nu^{2}(\kappa, \alpha, \beta)=\frac{1}{4}(\alpha+\beta+2 \kappa)(2+\alpha+\beta+2 \kappa),
$$

computed in Appendix A. In terms of the constituent quark masses the longitudinal mode expansion (42) can be expressed as

$$
\begin{aligned}
M_{\|}^{2}= & \left(m_{q}+m_{\bar{q}}\right)^{2}+\sigma\left(m_{q}+m_{\bar{q}}\right) \\
& +\sum_{\kappa=1}^{\infty} C_{\kappa}^{2}\left[\left(2 \kappa \sigma\left(m_{q}+m_{\bar{q}}\right)+\kappa(\kappa+1) \sigma^{2}\right] .\right.
\end{aligned}
$$

The rapid convergence of (44) in the basis function (38) found in [27,30], and discussed below in Sec. V, effectively ensures that the longitudinal mass squared does not grow as $\kappa^{2}$ for large $\kappa$, which would contradict linear Regge behavior.

\section{A. Chiral symmetry breaking}

The chiral limit follows directly from (44) since all the coefficients $C_{\kappa}$ vanish for $\kappa \neq 0$ in this limit [see (A7)]. We obtain for the pion mass

$$
M_{\pi}^{2}=\sigma\left(m_{u}+m_{d}\right)+\mathcal{O}\left(\left(m_{u}+m_{d}\right)^{2}\right),
$$

in the limit $m_{u}, m_{d} \rightarrow 0$. It has the same linear GMOR dependence in the quark mass [39] 
$M_{\pi}^{2} f_{\pi}^{2}=-\frac{1}{2}\left(m_{u}+m_{d}\right)\langle\bar{u} u+\bar{d} d\rangle+\mathcal{O}\left(\left(m_{u}+m_{d}\right)^{2}\right)$.

Formally the longitudinal coupling $\sigma$ sigma is given by $\sigma=-\langle\bar{\psi} \psi\rangle / f_{\pi}^{2}$, where the vacuum condensate $\langle\bar{\psi} \psi\rangle \equiv$ $\frac{1}{2}\langle\bar{u} u+\bar{d} d\rangle$ plays the role of a chiral order parameter. The same linear dependence in (45) arises for the $(3+1)$ effective LF Hamiltonian, since the constraints from the superconformal algebra require that the contribution to the pion mass from the transverse LF dynamics is identically zero [8].

Comparison with (36) leads to

$$
\sigma=g \sqrt{\frac{\pi N_{C}}{3}}=\text { const }
$$

since $g$ scales as $g \sim 1 / \sqrt{N_{C}}$ and chiral logarithms are suppressed at $N_{C} \rightarrow \infty$. Both (45) and (36) receive identical contributions from the potential and kinetic energy terms in agreement with the virial theorem.

\section{B. Heavy quark masses}

It is well known [47] that for heavy mesons the product $\sqrt{M} f_{M}$, where $f_{M}$ is the meson decay constant, approaches, up to logarithmic terms, a finite value $\sqrt{M} f_{M} \rightarrow C$, in agreement with the heavy-quark effective theory (HQET) result [48]. In the LF holographic context this means that the confinement scale $\lambda_{Q}$ has to increase with increasing quark mass $m_{Q}$. In the limit of heavy quarks the meson mass equals the sum of quark masses [cf., Eq. (44)], which requires that the confining scale is proportional to the mass of the heavy meson $M_{M}[13,28]$

$$
\sqrt{\lambda_{Q}}=C \sqrt{M_{Q}}
$$

In [15] the value $C=0.49 \pm 0.02 \mathrm{GeV}^{1 / 2}$ was found by fitting (48) in the mass interval $2 \leq M_{M} \leq 10 \mathrm{GeV}$ as shown in Fig. 1. Thus

$$
\sigma \simeq \frac{\lambda_{Q}}{M_{M}} \simeq C^{2} \simeq 0.24 \mathrm{GeV}
$$

We fix the longitudinal coupling $\sigma$ to the value found in [15], namely $\sigma=0.24 \mathrm{GeV}$. We have kept the same mass dependence of the transverse scale $\lambda$ (Fig. 1) since it reproduces quite well the slopes of Regge families across the hadron spectrum. We have extrapolated the value of $\sigma$ to the light quark mass domain, where we have no guidance from HQET, by assuming that $\sigma$ remain approximately constant, a result supported by the large $N_{C}$ result (47) and consistency with the chiral limit.

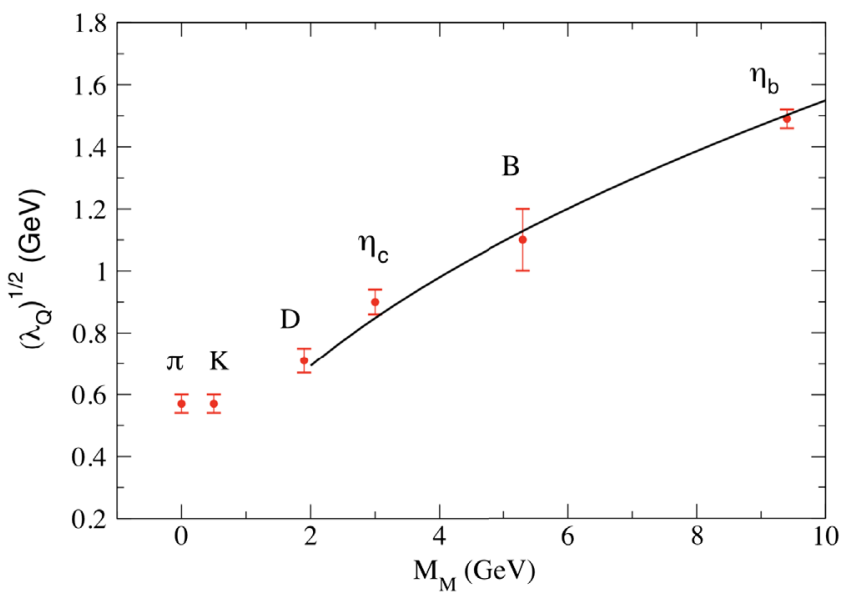

FIG. 1. Fitted values of $\sqrt{\lambda_{Q}}$ as a function of the mass of the lowest meson state $M_{M}$ on the Regge trajectory from Ref [15]. The solid line is the fit from Eq. (48).

\section{NUMERICAL RESULTS}

Having fixed the value of the longitudinal confinement scale $\sigma$ by the rate of change of the transverse confinement scale $\lambda$, we need to determine the value of the effective quark masses in (44) to compute the longitudinal mass contribution $M_{\|}^{2}$ to the total hadron mass. Thus, we determine the effective light quark masses $m_{u}$ and $m_{d}$ from the measured pion mass and the strange quark mass, $m_{s}$, from the kaon mass using (44): The value of the $\phi(1020)$ mass is then a prediction. Notice that the $\phi(1020)$ vector meson also has the transverse mass component (29), $M_{\perp}=\sqrt{2 \lambda}$, from the spin-spin interaction in supersymmetric LF holographic QCD $[9,36]$ with $\sqrt{\lambda}=0.523 \pm 0.024 \mathrm{GeV}$.

We show in Table I the lowest order coefficients in the expansion (40). The results for the light meson masses in Fig. 2 correspond to the values $m_{u}=m_{d}=28 \mathrm{MeV}$ and $m_{s}=326 \mathrm{MeV}$. The quark masses $m_{q}$ and $m_{\bar{q}}$ in (13) are effective quark masses from the renormalization due to the reduction of higher Fock states as functionals of the valence state [49], not the current quark masses in the QCD Lagrangian. The actual value of $\sigma$ used here is determined from the heavy quark mass constraint (39) to have a continuous description across the light and heavy scales. If instead $\sigma$ is determined by the current quark masses one obtains the value $\sigma \simeq 3 \mathrm{GeV}$, namely an order of magnitude

TABLE I. Lowest order coefficients $C_{\kappa}$ in the expansion (40).

\begin{tabular}{cccccccc}
\hline \hline & $\kappa=0$ & $\kappa=1$ & $\kappa=2$ & $\kappa=3$ & $\kappa=4$ & $\kappa=5$ & $\kappa=6$ \\
\hline$C(u \bar{d})$ & 0.998 & 0 & 0.055 & 0 & 0.010 & 0 & -0.003 \\
$C(u \bar{s})$ & 0.967 & -0.231 & 0.100 & -0.006 & -0.009 & 0.013 & -0.016 \\
$C(s \bar{s})$ & 0.998 & 0 & 0.038 & 0 & -0.045 & 0 & -0.024 \\
$C(u \bar{c})$ & 0.958 & -0.267 & 0.097 & -0.012 & -0.003 & 0 & -0.007 \\
$C(c \bar{c})$ & 0.999 & 0 & 0.016 & 0 & -0.020 & 0 & -0.003 \\
\hline \hline
\end{tabular}




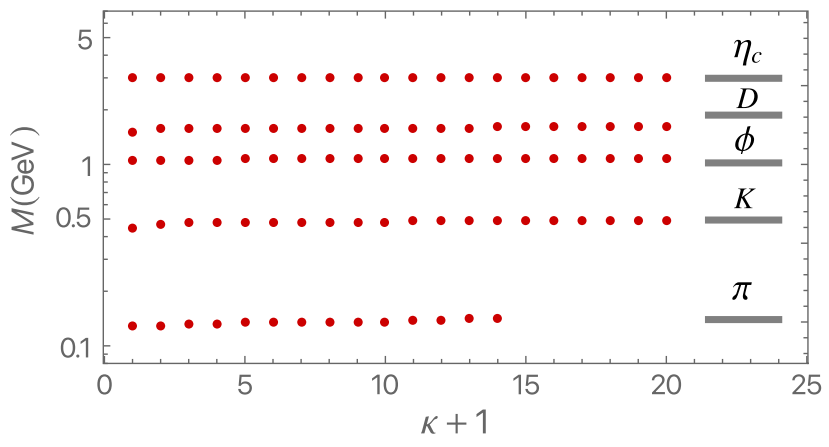

FIG. 2. Numerical evaluation of ground state meson masses from (44). The horizontal grey lines in the figure are the observed masses [50] also given in Table II for comparison.

larger than the transverse scale $\sqrt{\lambda} \simeq 0.5 \mathrm{GeV}$, yielding an unphysical spectrum.

Meson masses are determined from the stability plateau in Fig. 2. For light quark masses, contributions above $\kappa_{\max } \simeq 20$ introduce large uncertainties from highly oscillatory integrands. In Fig. 3 we show the effect of the strong oscillations of the Jacobi polynomials at large $\kappa$ [51] by examining the dependence of the pion mass, $M_{\pi}$, for quark masses in the interval $m_{q}=28 \mathrm{MeV}$ to $m_{q}=28 \times$ $10^{-8} \mathrm{MeV}$ for fixed $\sigma$.

The meson distribution amplitudes (DAs) [52]

$$
\phi_{M}(x) \sim X(x)=\sqrt{x(1-x)} \chi(x)
$$

for the pion, kaon, $\mathrm{D}$, and $J / \Psi$ mesons are shown in Fig. 4. Due to the rapid convergence of the exponential wave function in the basis expansion (40), very few modes are required to reproduce the invariant mass LFWF. The DAs predicted by HLFQCD at the initial nonperturbative scale should then be evolved to the relevant scale using the

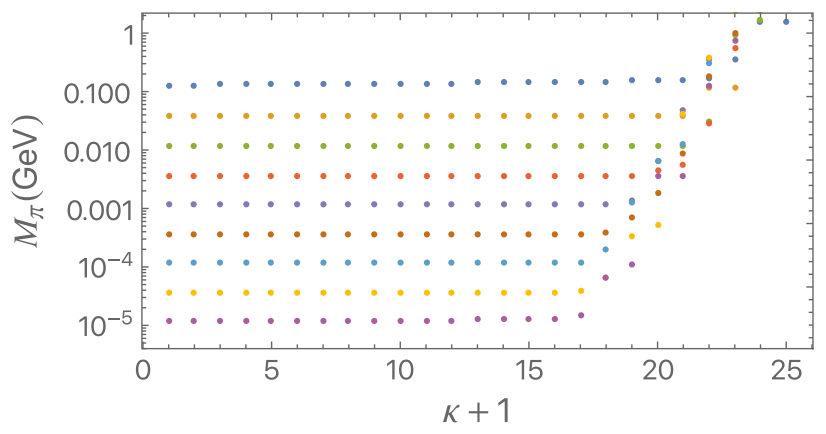

FIG. 3. The numerical evaluation of the pion mass for quark masses in the range $m_{q}=28 \mathrm{MeV}$ (upper blue dotted line) to $m_{q}=28 \times 10^{-8} \mathrm{MeV}$ (lower mauve dotted line), for fixed $\sigma$, manifests the numerical spurious divergence from highly oscillatory integrands at large $\kappa$, in sharp contrast with the exact chiral result (45).
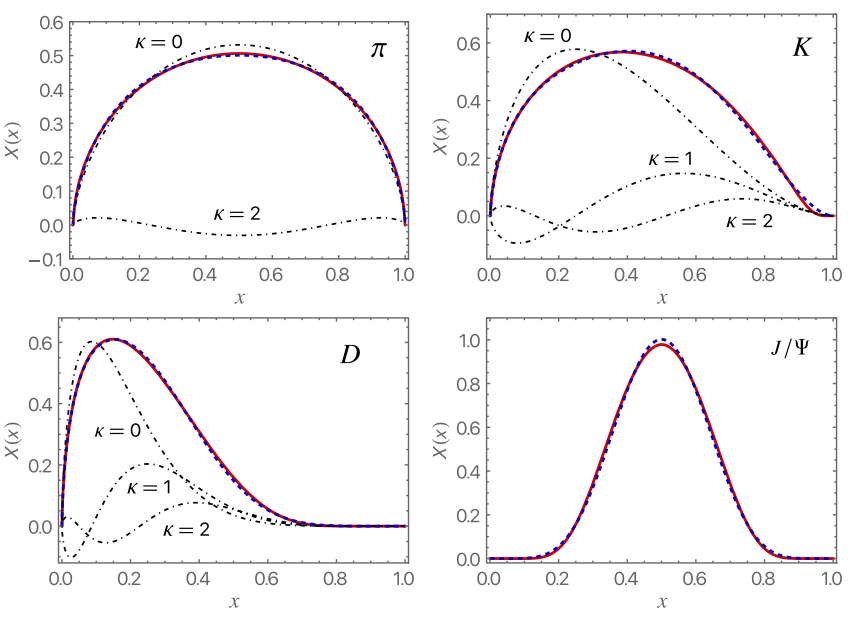

FIG. 4. Light-front distribution amplitudes $X(x)$ for the $\pi, K, D$ and $J / \Psi$ mesons: the red curve is the invariant mass result, dot dashed black curves are individual modes in the expansion (40), dashed blue curve represents the sum of modes in the figure. Notice that the $J / \Psi$ result is well described by the zero-order Jacobi Polynomial.

ERBL equation [52-54]. The Dyson-Schwinger results for the pion DA [55] are very similar to the chiral limit result $\phi_{\pi}(x) \sim \sqrt{x(1-x)}$ from LF holographic mapping [32].

Finally, we can extend our analysis to the heavy quark sector provided that longitudinal and transverse dynamics can be separated to a good approximation. In contrast with light quark masses $m_{q}, m_{\bar{q}} \ll \sigma, q=u, d$, most of the hadron mass in the heavy sector, $m_{Q}, m_{\bar{Q}} \gg \sigma$, comes from quark masses. The expansion coefficients for the wave function (40) for the $u c$ and $c c$ mesons are shown in Table I. We determine the effective charm quark mass from the $\eta_{c}$ using (44) and compute, for example, the mass of the $D$ meson as a prediction. We find for $M_{D}$ a value within $14 \%$ of its measured value for $m_{c} \simeq 1.4 \mathrm{GeV}$ (Table II). Our results do not include the negative contribution from one-gluon exchange for the small size double-heavy mesons $[28,30]$ which gives a larger value for the extracted charmed quark mass, therefore a value for the $D$ meson mass closer to its experimental value.

TABLE II. Comparison of meson mass results in Fig. 2 (2nd column) with observed values from [50] (3rd column). Onegluon exchange corrections are not included for the charmonium and bottonium.

\begin{tabular}{lcc}
\hline \hline & $M(\mathrm{GeV})$ & $M_{\text {data }}(\mathrm{GeV})$ \\
\hline$\pi^{ \pm}$ & 0.140 & 0.140 \\
$K^{ \pm}$ & 0.490 & 0.494 \\
$\phi(1020)$ & 1.067 & 1.019 \\
$D^{ \pm}$ & 1.602 & 1.870 \\
$\eta_{c}(1 S)$ & 2.978 & 2.984 \\
\hline \hline
\end{tabular}




\section{CONCLUSIONS AND OUTLOOK}

The light-front semiclassical approximation described in this article incorporates the confinement strength in the longitudinal direction as well as the effective scale of chiral symmetry breaking as different manifestations of the same underlying dynamics. As such, it accounts for most of the meson mass, consistent with the GMOR relation. Following [27] we have separated LF longitudinal and transverse dynamics and adopted from [30] a potential which generates a convenient basis function in the longitudinal direction. It is responsible for breaking the chiral symmetry and restoring rotational symmetry in the limit of heavy quark masses. In practice, it allows us to reduce the highly complex and non-local four-dimensional LF QCD Hamiltonian to a set of two independent second order differential equations, consistent with the local structure of semiclassical AdS equations [56,57].

The mass dependence of the transverse scale $\lambda$ found in $[13,15]$ is unchanged, therefore the Regge slopes are unmodified. The changes introduced by the new approach primarily modify the lowest meson state in each Regge trajectory, and therefore the value of the extracted effective quark masses. Our previous successful results for the Regge trajectories for light and heavy quark masses are basically unchanged.

The origin and physical interpretation of the longitudinal scale $\sigma$, which has the role of a condensate $\langle\bar{\psi} \psi\rangle$, remains to be explored, but as we have shown in (47), it is related to the dimensionful constant $g$ in $\mathrm{QCD}(1+1)$ at large $N_{C}$. In lattice QCD the structure of the vacuum is sampled in the Euclidean region where non-trivial gauge field configurations provide a mechanism for symmetry breaking through the Banks-Casher relation, $\langle\bar{\psi} \psi\rangle=-\pi \rho(0)$, with $\rho(0)$ the density or Dirac-zero modes $[58,59]$. However, the relation between chiral symmetry breaking and confinement has remained elusive. In this context, it has been argued that the chiral condensate, usually viewed as a constant mass scale which fills all spacetime, is instead contained within hadrons, therefore a property of hadron dynamics [60,61].

The fact that the nonzero pion mass is a consequence of longitudinal LF confinement is a remarkable result. One would expect, for example, from the two-dimensional 't Hooft [21] or Schwinger [22] models in light-front coordinates, that the pion mass from the mechanism of chiral symmetry breaking originates in the longitudinal component of the wave function [23], since the kinetic quark mass terms only depend on the longitudinal variable. However, this would not be the case if the transverse kinetic and potential energy of the pion would not exactly cancel as required by the superconformal structure of the transverse LF Hamiltonian. The pion plays a special role as the hadronic state of zero mass in the chiral limit. Since it does not have a baryonic partner, the pion breaks the mesonbaryon hadron supersymmetry [8]. In contrast, the proton mass (as well as the mass of radial and orbital hadron excited states) is generated by the addition of the transverse kinetic and transverse potential energy with a small contribution from the longitudinal dynamics, in agreement with the Regge phenomenology of the hadron mass spectrum.

\section{ACKNOWLEDGMENTS}

We thank Yang Li and James Vary for their collaboration in the early stages of this work and for reading our manuscript. We also thank Hans Guenter Dosch for critical remarks and Alexandre Deur, John Hiller, Valery Lyubovitsky, Gerald Miller and Ruben Sandapen for useful comments. The work of S. J. B. is supported in part by the Department of Energy, Contract No. DE-AC0276SF00515, SLAC-PUB-17593.

Note added.-We refer the reader to the related work by $\mathrm{Li}$ and Vary [62], submitted concurrently with this article, as well as to Ahmady et al. [63,64], submitted after ours. We thank Colin Weller and Gerald Miller for sharing with us their related upcoming article on confinement in twodimensional QCD.

\section{APPENDIX A: JACOBI POLYNOMIALS AND SOLUTION TO THE LONGITUDINAL HAMILTONIAN EQUATION}

The Jacobi polynomials $P_{\kappa}^{(\alpha, \beta)}(z)$ are solution of the differential equation

$$
\begin{aligned}
& (1-z)^{-\alpha}(1+z)^{-\beta} \frac{d}{d z}\left((1-z)^{\alpha+1}(1+z)^{\beta+1} \frac{d}{d z} u(z)\right) \\
& +\kappa(\kappa+a+b+1) u(z)=0,
\end{aligned}
$$

which is orthogonal in the interval $[-1,1]$ with weight $(1-z)^{\alpha}(1-z)^{\beta}$. Performing the change of variable $z=1-2 x$ we find

$$
\begin{gathered}
x^{-\alpha}(1-x)^{-\beta} \frac{d}{d x}\left(x^{\alpha+1}(1-x)^{\beta+1} \frac{d}{d x} u(x)\right) \\
+\kappa(\kappa+a+b+1) u(x)=0,
\end{gathered}
$$

with the solution $P_{\kappa}^{(\alpha, \beta)}(1-2 x)$ orthogonal in the interval $[0,1]$ with weight $x^{\alpha}(1-x)^{\beta}$.

Consider now the eigenvalue equation

$$
\begin{aligned}
& \left(-\frac{d}{d x}\left(x(1-x) \frac{d}{d x}\right)+\frac{1}{4}\left[\frac{\alpha^{2}}{x}+\frac{\beta^{2}}{1-x}\right]\right) v(x) \\
& =\nu^{2} v(x) .
\end{aligned}
$$

Writing $v(x)=x^{\alpha / 2}(1-x) x^{\beta / 2} w(x)$ and substituting in (A3) we find that $w(x)=P_{k}^{(\alpha, \beta)}(1-2 x)$. Therefore the normalized solution to (A3) 


$$
\chi_{\kappa}^{\alpha, \beta}(x)=N x^{\alpha / 2}(1-x)^{\beta / 2} P_{\kappa}^{(\alpha, \beta)}(1-2 x),
$$

with eigenvalues

$$
\nu^{2}=\frac{1}{4}(\alpha+\beta+2 \kappa)(2+\alpha+\beta+2 \kappa),
$$

and normalization

$$
N=\sqrt{1+\alpha+\beta+2 \kappa} \sqrt{\frac{\Gamma(1+\kappa) \Gamma(1+\alpha+\beta+\kappa)}{\Gamma(1+\alpha+\kappa) \Gamma(1+\beta+\kappa)}},
$$

given in terms of the Gamma function. If $\alpha=\beta=0$ the solution (A4) is reduced to

$$
\chi_{\kappa}^{0,0}(x)=\sqrt{1+2 \kappa} P_{\kappa}(1-2 x),
$$

where $P_{\kappa}(x)$ is a Legendre polynomial.

\section{APPENDIX B: HEAVY QUARK MASS LIMIT}

Rotational invariance of the oscillator potential in the nonrelativistic limit leads to a precise connection of the scale $\sigma$, of the longitudinal potential in Sec. III C, with the strength $\lambda$ of the transverse confinement potential in
Sec. III A, which sets the hadronic scale. To show this, consider the nonrelativistic limit $m_{q}, m_{\bar{q}} \rightarrow m_{Q}, m_{\bar{Q}} \gg k_{\perp}$, $k_{z}, \lambda \rightarrow \lambda_{Q}$ with

$$
x=\frac{m_{Q}+k_{z}}{m_{Q}+m_{\bar{Q}}}, \quad \bar{x}=\frac{m_{\bar{Q}}-k_{z}}{m_{Q}+m_{\bar{Q}}} .
$$

One finds the rotationally invariant potential

$$
U(r) \rightarrow V(r) \equiv \frac{U(r)}{m_{Q}+m_{\bar{Q}}}=\frac{1}{2} \mu \omega^{2} r^{2},
$$

and the constraint

$$
\omega=\sigma=\frac{\lambda_{Q}}{m_{Q}+m_{\bar{Q}}}
$$

in the limit of heavy quark masses where

$$
\mu=\frac{m_{Q} m_{\bar{Q}}}{m_{Q}+m_{\bar{Q}}}
$$

is the reduced mass of the heavy quark-antiquark system and $\mathbf{r}^{2}=\mathbf{b}_{\perp}^{2}+b_{z}^{2}[65]$.
[1] V. de Alfaro, S. Fubini, and G. Furlan, Conformal invariance in quantum mechanics, Nuovo Cimento Soc. Ital. Fis. 34A, 569 (1976).

[2] S. Fubini and E. Rabinovici, Superconformal quantum mechanics, Nucl. Phys. B245, 17 (1984).

[3] P. A. M. Dirac, Forms of relativistic dynamics, Rev. Mod. Phys. 21, 392 (1949).

[4] J. M. Maldacena, The large- $N$ limit of superconformal field theories and supergravity, Int. J. Theor. Phys. 38, 1113 (1999).

[5] G. F. de Téramond and S. J. Brodsky, Light-Front Holography: A First Approximation to QCD, Phys. Rev. Lett. 102, 081601 (2009).

[6] S. J. Brodsky, G. F. de Téramond, and H. G. Dosch, Threefold complementary approach to holographic QCD, Phys. Lett. B 729, 3 (2014).

[7] G. F. de Téramond, H. G. Dosch, and S. J. Brodsky, Baryon spectrum from superconformal quantum mechanics and its light-front holographic embedding, Phys. Rev. D 91, 045040 (2015).

[8] H. G. Dosch, G. F. de Téramond, and S. J. Brodsky, Superconformal baryon-meson symmetry and light-front holographic QCD, Phys. Rev. D 91, 085016 (2015).

[9] S. J. Brodsky, G. F. de Téramond, H. G. Dosch, and J. Erlich, Light-front holographic QCD and emerging confinement, Phys. Rep. 584, 1 (2015).
[10] S. J. Brodsky, G. F. de Téramond, and H. G. Dosch, Lightfront holography and supersymmetric conformal algebra: A novel approach to hadron spectroscopy, structure, and dynamics, arXiv:2004.07756.

[11] G. F. de Téramond, H. G. Dosch, and S. J. Brodsky, Kinematical and dynamical aspects of higher-spin bound-state equations in holographic QCD, Phys. Rev. D 87, 075005 (2013).

[12] H. G. Dosch, G. F. de Téramond, and S. J. Brodsky, Supersymmetry across the light and heavy-light hadronic spectrum, Phys. Rev. D 92, 074010 (2015).

[13] H. G. Dosch, G. F. de Téramond, and S. J. Brodsky, Supersymmetry across the light and heavy-light hadronic spectrum II, Phys. Rev. D 95, 034016 (2017).

[14] M. Nielsen and S. J. Brodsky, Hadronic superpartners from a superconformal and supersymmetric algebra, Phys. Rev. D 97, 114001 (2018).

[15] M. Nielsen, S. J. Brodsky, G. F. de Téramond, H. G. Dosch, F. S. Navarra, and L. Zou, Supersymmetry in the doubleheavy hadronic spectrum, Phys. Rev. D 98, 034002 (2018).

[16] R. S. Sufian, G. F. de Téramond, S. J. Brodsky, A. Deur, and H. G. Dosch, Analysis of nucleon electromagnetic form factors from light-front holographic QCD: The spacelike region, Phys. Rev. D 95, 014011 (2017).

[17] G. F. de Téramond, T. Liu, R. S. Sufian, H. G. Dosch, S. J. Brodsky, and A. Deur, Universality of Generalized Parton 
Distributions in Light-Front Holographic QCD, Phys. Rev. Lett. 120, 182001 (2018).

[18] T. Liu, R. S. Sufian, G. F. de Téramond, H. G. Dosch, S. J. Brodsky, and A. Deur, Unified Description of Polarized and Unpolarized Quark Distributions in the Proton, Phys. Rev. Lett. 124, 082003 (2020).

[19] S. J. Brodsky, G. F. de Téramond, and A. Deur, Nonperturbative QCD coupling and its $\beta$ function from light-front holography, Phys. Rev. D 81, 096010 (2010).

[20] A. Deur, S. J. Brodsky, and G. F. de Téramond, Connecting the hadron mass scale to the fundamental mass scale of quantum chromodynamics, Phys. Lett. B 750, 528 (2015); On the interface between perturbative and nonperturbative QCD, Phys. Lett. B 757, 275 (2016).

[21] G. 't Hooft, A two-dimensional model for mesons, Nucl. Phys. B75, 461 (1974).

[22] H. Bergknoff, Physical particles of the massive Schwinger model, Nucl. Phys. B122, 215 (1977).

[23] A. R. Zhitnitsky, On chiral symmetry breaking in QCD in two-dimensions $\left(N_{c} \rightarrow \infty\right)$, Phys. Lett. 165B, 405 (1985).

[24] K. Hornbostel, S. J. Brodsky, and H. C. Pauli, Light-conequantized QCD in $(1+1)$-dimensions, Phys. Rev. D 41, 3814 (1990).

[25] Y. Mo and R. J. Perry, Basis function calculations for the massive Schwinger model in the light-front Tamm-Dancoff approximation, J. Comput. Phys. 108, 159 (1993).

[26] S. J. Brodsky and G. F. de Téramond, AdS/CFT and lightfront QCD, Subnuclear series 45, 139 (2009).

[27] S. S. Chabysheva and J. R. Hiller, Dynamical model for longitudinal wave functions in light-front holographic QCD, Ann. Phys. (Amsterdam) 337, 143 (2013).

[28] T. Gutsche, V. E. Lyubovitskij, I. Schmidt, and A. Vega, Chiral symmetry breaking and meson wave functions in soft-wall AdS/QCD, Phys. Rev. D 87, 056001 (2013).

[29] A. P. Trawiński, S. D. Głazek, S. J. Brodsky, G. F. de Téramond, and H. G. Dosch, Effective confining potentials for QCD, Phys. Rev. D 90, 074017 (2014).

[30] Y. Li, P. Maris, X. Zhao, and J. P. Vary, Heavy quarkonium in a holographic basis, Phys. Lett. B 758, 118 (2016).

[31] In the usual formulation of bottom-up holographic models one identifies quark mass and chiral condensates as coefficients of a scalar background field $X_{0}(z)$ in AdS space. See: J. Erlich, E. Katz, D. T. Son, and M. A. Stephanov, QCD and Holographic Model of Hadrons, Phys. Rev. Lett. 95, 261602 (2005); L. Da Rold and A. Pomarol, Chiral symmetry breaking from five dimensional spaces, Nucl. Phys. B721, 79 (2005).

[32] S. J. Brodsky and G. F. de Téramond, Hadronic Spectra and Light-Front Wave Functions in Holographic QCD, Phys. Rev. Lett. 96, 201601 (2006).

[33] For a recent overview see, R. Sandapen, An overview of light-front holography, Proc. Sci., LC2019 (2020) 011 [arXiv:2001.03479], and references therein.

[34] W. I. Weisberger, Partons, electromagnetic mass shifts, and the approach to scaling, Phys. Rev. D 5, 2600 (1972).

[35] A. Karch, E. Katz, D. T. Son, and M. A. Stephanov, Linear confinement and AdS/QCD, Phys. Rev. D 74, 015005 (2006).

[36] S. J. Brodsky, G. F. de Téramond, H. G. Dosch, and C. Lorcé, Universal effective hadron dynamics from superconformal algebra, Phys. Lett. B 759, 171 (2016).
[37] L. Zou and H. G. Dosch, A very practical guide to light front holographic QCD, arXiv:1801.00607.

[38] S. J. Brodsky, H. C. Pauli, and S. S. Pinsky, Quantum chromodynamics and other field theories on the light cone, Phys. Rep. 301, 299 (1998).

[39] M. Gell-Mann, R. J. Oakes, and B. Renner, Behavior of current divergences under $S U(3) \times S U(3)$, Phys. Rev. 175, 2195 (1968).

[40] J. P. Vary, H. Honkanen, J. Li, P. Maris, S. J. Brodsky, A. Harindranath, G. F. de Téramond, P. Sternberg, E. G. Ng, and C. Yang, Hamiltonian light-front field theory in a basis function approach, Phys. Rev. C 81, 035205 (2010).

[41] Y. Li, P. W. Wiecki, X. Zhao, P. Maris, and J. P. Vary, Introduction to basis light-front quantization approach to QCD bound state problems, arXiv:1311.2980.

[42] Y. Li, P. Maris, and J. P. Vary, Quarkonium as a relativistic bound state on the light front, Phys. Rev. D 96, 016022 (2017).

[43] C. Mondal, S. Xu, J. Lan, X. Zhao, Y. Li, D. Chakrabarti, and J.P. Vary, Proton structure from a light-front Hamiltonian, Phys. Rev. D 102, 016008 (2020).

[44] W. Qian, S. Jia, Y. Li, and J. P. Vary, Light mesons within the basis light-front quantization framework, Phys. Rev. C 102, 055207 (2020).

[45] A. B. Sheckler and G. A. Miller, Mystery of Bloom-Gilman duality: A light-front holographic QCD perspective, Phys. Rev. D 103, 096018 (2021).

[46] M. Kreshchuk, S. Jia, W. M. Kirby, G. Goldstein, J. P. Vary, and P. J. Love, Simulating hadronic physics on NISQ devices using basis light-front quantization, Phys. Rev. A 103, 062601 (2021).

[47] E. V. Shuryak, Hadrons containing a heavy quark and QCD sum rules, Nucl. Phys. B198, 83 (1982).

[48] N. Isgur and M. B. Wise, Spectroscopy with Heavy Quark Symmetry, Phys. Rev. Lett. 66, 1130 (1991).

[49] H. C. Pauli, On confinement in a light-cone Hamiltonian for QCD, Eur. Phys. J. C 7, 289 (1999).

[50] P. A. Zyla et al. (Particle Data Group), Review of particle physics, Prog. Theor. Exp. Phys. 2020, 083C01 (2020).

[51] Gábor Szegő, Orthogonal Polynomials, 4th ed. (American Mathematical Society, Providence, 1975).

[52] G. P. Lepage and S. J. Brodsky, Exclusive processes in quantum chromodynamics: Evolution equations for hadronic wave functions and the form-factors of mesons, Phys. Lett. 87B, 359 (1979).

[53] A. V. Efremov and A. V. Radyushkin, Factorization and asymptotic behavior of pion form factor in QCD, Phys. Lett. 94B, 245 (1980).

[54] S. J. Brodsky, F. G. Cao, and G. F. de Téramond, Evolved QCD predictions for the meson-photon transition form factors, Phys. Rev. D 84, 033001 (2011).

[55] C. D. Roberts, D. G. Richards, T. Horn, and L. Chang, Insights into the emergence of mass from studies of pion and kaon structure, Prog. Part. Nucl. Phys. 120, 103883 (2021), and references therein.

[56] R. Narayanan and H. Neuberger, The quark mass dependence of the pion mass at infinite $N$, Phys. Lett. B 616, 76 (2005). 
[57] E. Katz and T. Okui, The 't Hooft model as a hologram, J. High Energy Phys. 01 (2009) 013.

[58] T. Banks and A. Casher, Chiral symmetry breaking in confining theories, Nucl. Phys. B169, 103 (1980).

[59] See, for example, C. McNeile, A. Bazavov, C. T. H. Davies, R. J. Dowdall, K. Hornbostel, G. P. Lepage, and H. D. Trottier, Direct determination of the strange and light quark condensates from full lattice QCD, Phys. Rev. D 87, 034503 (2013), and references therein.

[60] S. J. Brodsky, C. D. Roberts, R. Shrock, and P. C. Tandy, Confinement contains condensates, Phys. Rev. C 85, 065202 (2012).

[61] P. D. Mannheim, P. Lowdon, and S. J. Brodsky, Structure of light-front vacuum sector diagrams, Phys. Lett. B 797, 134916 (2019).

[62] Y. Li and J. P. Vary, Light-front holography with chiral symmetry breaking, arXiv:2103.09993.
[63] M. Ahmady, H. Dahiya, S. Kaur, C. Mondal, R. Sandapen, and N. Sharma, Extending light-front holographic QCD using the 't Hooft Equation, Phys. Lett. B 823, 136754 (2021).

[64] M. Ahmady, S. Kaur, S. L. MacKay, C. Mondal, and R. Sandapen, Hadron spectroscopy using the light-front holographic Schrödinger equation and the 't Hooft equation, Phys. Rev. D 104, 074013 (2021).

[65] The longitudinal spatial variable $\tilde{z}$ conjugate to the longitudinal momentum $x, \tilde{z} \rightarrow i \partial_{x}$, is the frame-independent Ioffe coordinate of Miller and Brodsky and $b_{z}$ is the canonical conjugate to $k_{z}, b_{z} \rightarrow i \partial_{k_{z}}$. See: G. A. Miller and S. J. Brodsky, Frame-independent spatial coordinate $\tilde{z}$ : Implications for light-front wave functions, deep inelastic scattering, light-front holography, and lattice QCD calculations, Phys. Rev. C 102, 022201 (2020). 